Pacific Journal of Mathematics

MAXIMUM MODULUS THEOREMS FOR ALGEBRAS O 


\title{
MAXIMUM MODULUS THEOREMS FOR ALGEBRAS OF OPERATOR VALUED FUNCTIONS
}

\author{
KENNETH O. LELAND
}

Let $F$ be a family of functions on subsets of a real Euclidean space $E$ into a commutative subalgebra with identity $T_{0}$ of the algebra $T$ of linear transformations of $E$ into itself. If a suitable integration condition, motivated by Morera's theorem in complex function theory is placed on the elements of $F, F$ becomes an algebra of "integrable" functions which can be realized as the derivatives of transformations of $E$ into itself. It is asked what properties of the algebra of complex analytic functions from the complex plane $K$ into $K$ are satisfied by such algebras $F$. Simple examples show that analyticity and even differentiability are lost. However various forms of the maximum modulus theorem are still satisfied. Three such theorems are presented here:

(A) If commutivity of $T_{0}$ is replaced by the requirement that the elements of $T_{0}$ are "orientation preserving" then the elements of $F$ are maximized on the boundary of a sphere.

(B) There exists $N>0$, such that for all $f \in F$,

$$
\bar{U}=\{t \in E ;\|t\| \leqq 1\} \subseteq \text { domain } f, x \in \bar{U} \text {, }
$$

implies

$$
\|f(x)\| \leqq N \sup \{\|f(t)\| ;\|t\|=\mathbf{1}\} .
$$

(C) For all $f \in F, \bar{U} \subseteq$ domain $f, x \in \bar{U}$, implies

$$
\|f(x)\|_{s} \leqq \sup \left\{\|f(t)\|_{s} ;\|t\|=1\right\},
$$

where for $A \in T_{0},\|A\|_{s}$ is the spectral norm of $A$.

1. Introduction. The theory of complex valued integrable functions was developed by Heffter [1], Macintyre and Wilbur [6], and this author [4]. The generalization to the operator valued case was introduced in [5].

The first two results of this paper employ degree theoretic methods from algebraic topology as developed in [3] to obtain similar results. The methods of [3] represent a generalization to higher dimensional speces of methods of G. T. Whyburn [8] in the plane, employed by him to handle complex analytic functions. The third result follows from a construction of the spectral norm of an operator employing irreducible subspaces invariant under a family of operators.

Let $K$ denote the complex plane. For $a \in K$, set $A_{a}(z)=a z$ for 
all $z \in K$. Then $A_{a}$ is a bounded linear transformation of $K$ thought of as a real Euclidean space $E_{2}$ into itself. Set $T_{2}^{\prime}=\left\{A_{a} ; a \in K\right\}$. Let $f$ be a continuous function on an open set $S \subseteq E_{2}$ into the space $B_{2}$ of bounded linear transformations of $E_{2}$ into itself, and let $P$ be a path (rectifiable arc) with endpoints $\alpha$ and $\beta$. Then for any subdivision $\alpha=x_{0}<\cdots<x_{n+1}=\beta$ of $P$, a Riemann sum, the vector $R=\sum_{i=1}^{n} f_{z_{i}}\left(z_{i+1}-z_{i}\right)$ can be formed. If range $f$ lies in $T_{2}^{\prime}$, then for $z \in S, f_{z}=f(z)=A_{\dot{\phi}(z)}$ for some $\dot{\phi}(z) \in K$, and we may write

$$
R=\sum_{i=0}^{n} \phi\left(z_{i}\right)\left(z_{i+1}-z_{i}\right) \text {. }
$$

Taking the limit as the norm of the subdivision defining $R$ approaches zero, we obtain the vector $\theta=\int_{\alpha P}^{\beta} f(z) d z=\int_{\alpha P}^{\beta} f_{z}(d z)$. If range $f \subseteq T_{2}^{\prime}$, we can interpret $\theta$ as the complex number $\int_{\alpha P}^{\beta} \phi(z) d z$.

$f$ is said to be integrable if for closed paths (rectifiable simple closed curves) $C \subseteq S$, we have $\int_{C} f(z) d z=\int_{C} f_{z}(d z)=0$. If range $f \subseteq T_{2}^{\prime}$, then $\int_{C} \phi(z) d z=0$ for all closed paths $C \leqq S$, and by Morera's theorem $\phi$ is analytic; consequently, $f$ is itself Frechét differentiable, where $f_{z}^{\prime}$ is a linear transformation of $E_{2}$ into $B_{2}$ for $z \in S$.

The general case studied in this paper is obtained by replacing $E_{2}$ by an arbitrary real Euclidean space $E$ of dimension $p, p>1$. Let $T$ be a commutative subalgebra of the Banach algebra of bounded linear transformations of $E$ into $E$ and let $F$ be the family of continuous integrable functions on open subsets of $E$ into $T$.

Let $f \in F, S=$ domain $f$ simply connected. Let $z_{0} \in S$ and for $z \in S$, set $g(z)=\int_{z_{0}}^{z} f(z) d z$. Then $g$ maps $S$ into $E$, and for $z \in S$, the Frechét derivative $g_{z}^{\prime}$ of $g$ at $z$ is the operator $f(z)=f_{z}$ of $T$.

In [5], employing arguments reminiscent of the proof of the Cauchy-Goursat theorem, it is shown that the family of continuous integrable functions on a simply connected subset of $E$ into $T$ form an algebra.

In [5] differentiability and analyticity of integrable functions is discussed. Simple examples of integrable but nondifferentiable functions are given. Since the context of [5] and this paper is a real variable context a definition of analyticity motivated by Schwarz's lemma was employed [2].

The development of this paper is no way affected if the only paths of integration permitted are those formed from straight line segments or ares of circles.

2. Notation and definitions. Let $\omega$ denote the positive integers. 
If $Z$ is a Banach space, $\hat{o}>0, x \in Z$, set $U_{x}(\delta)=\{t \in Z ;\|t-x\|<\hat{o}\}$, $U(\delta)=U_{0}(\hat{o})$, and $U=U_{0}(1)$; and set $V_{x}(\delta)=\{t \in Z ;\|t-x\|=\hat{o}\}$, $V(\delta)=V_{0}(\delta)$, and $V=V_{0}(1)$. If $f$ is a function with domain $S$ and $H \subseteq E$, then the restriction $f \mid H$ of $f$ to $H$ is the function $g$ with domain $H \cap S$ such that $g(x)=f(x)$ for all $x \in H \cap S$. For $H$ a subspace of $E$ and $G$ a family of functions defined on subsets of $E, F \mid H$ denotes the family $\left\{f^{\prime} \mid H ; f^{\prime} \in F\right\}$.

An element $A$ of $B$ is said to be orientation preserving if $A$ inverts and if the degree $\mu(A)$ of $A$ is one (or equivalently if the determinant associated with $A$ is positive). A collection of operators $Z$ of $B$ is said to be an orientation preserving family if for all $A \in Z$, $r \geqq 0$, we have that if $A+r e$ inverts, then $A+r e$ is orientation preserving.

For $A \in B$, set $\|A\|_{s}=\lim \sup _{n \rightarrow \infty}\left\|A^{n}\right\|^{1 / n}$. It shall be shown in $\S 7$, that $\|\cdot\|_{i}$ is a multiplicative semi-norm.

3. Statement of main results. Let $\rho>1$, and let $f$ be an integrable function on $U(\rho)$ into $B$. Then the principle results of this paper are:

(A) The strong maximum modulus theorem which states that if range $f$ lies in an orientation preserving family $Z$ of $B, Z$ a linear subspace of $B$, then for all $x \in U$,

$$
\|f(x)\| \leqq M=\sup \{\|f(t)\| ; t \in V\} .
$$

(B) The weak maximum modulus theorem which states that there exists $N>0$ such that if $f \in F$, then for all $x \in U$,

$$
\|f(x)\| \leqq N M \text {. }
$$

(C) The maximum spectral norm theorem which states that if $f \in F$, then for $x \in U$,

$$
\|f(x)\|_{s} \leqq \sup \left\{\|f(t)\|_{s} ; t \in V\right\} .
$$

We note that for $E$ and $T$ isomorphic and isometric to $K$, that $T$ is an orientation preserving family and that in this case for $Z=T$, $(A)$ and $(C)$ reduce to the standard maximum modulus theorem of complex function theory.

4. Preliminaries. We shall need two lemmas from [5].

A subspace $W \subseteq E$ is said to be invariant if $T(W) \subseteq W$. Let $\mathscr{C}$ be the family of all nontrivial irreducible invariant subspaces of $E$, i.e., the family of all invariant subspaces $H \subseteq E, H \neq\{0\}$, such that $H$ contains no proper invariant subspace $H_{0} \neq\{0\}$. 
LemmA 4.1. For $H \in \mathscr{L}, T_{0}=T \mid H$ is a field with the same dimension as $H$.

We observe from the fundamental theorem of algebra that $T_{0}$ is isomorphic (but not necessarily isometric) to $R$ or $K$.

Proof. Let $A \in T_{0}, A \neq 0$. Then $A$ is one-to-one on $H$. Indeed, for $D \in T$, setting $D_{0}=D \mid H$, we have

$$
D[A(H)]=D_{0}[A(H)]=A\left[D_{0}(H)\right]=A[D(H)] \subseteq A(H),
$$

and hence $A(H)$ is an invariant subspace of $H$; and consequently, since $H$ is irreducible, $A(H)=H$.

Let $x \in H, x \neq 0$, and set $\theta(A)=A(x)$ for all $A \in T_{0}$. Now range $\theta=\{A(x) ; A \in T\}$ is clearly an invariant subspace of $H$ and hence range $\theta=H$. Suppose for $A, B \in T_{0}, \theta(A)=\theta(B)$. Then $A(x)=B(x)$ and $(A-B)(x)=0$. Since $A-B$ is not one-to-one on $H, A-B=0$, and thus $\theta$ is an isomorphism of $T_{0}$ onto $H$.

Let $A \in T_{0}$. Since $A(H)=H$, there exists $y \in H$, such that $A(y)=x$. Now there exists $\alpha \in T_{0}$ such that $\theta(\alpha)=y$. Then $\alpha(x)=y$ and $\theta(A \alpha)=(A \alpha)(x)=A[\alpha(x)]=A(y)=x$. Since $\theta(e)=x$, we have $A \alpha=e$ and $\alpha=A^{-1}$, and thus $T_{0}$ is a field.

LEMma 4.2. Let $f$ be an integrable function on $U$ into $B$, and $S$ and $W$ subspaces of $E$ such that for $x \in U, t \in S, f(x)(t)$ lies in $W$. Then for $x, y \in U$ such that $y-x$ lies in $S$, we have

$$
[f(x)-f(y)](t) \in W \quad \text { for all } t \in E .
$$

Proof. For $x \in U$, set $g(x)=\int_{0}^{x} f(z) d z$. Let $x, y \in U$ such that $x \neq y$ and $y-x \in S$, and let $x=x_{0}<\cdots<x_{n+1}=y, n \in \omega$, be a subdivision of the interval $[x, y]$ of $E$. Then for

$$
i=0,1, \cdots, n, \Delta x_{i}=x_{i+1}-x_{i} \in S,
$$

and hence $f\left(x_{i}\right)\left(\Delta x_{i}\right) \in W$. Thus the Riemann sum $\sum_{0}^{n} f\left(x_{i}\right)\left(\Delta x_{i}\right) \in W$. Hence $\int_{x}^{y} f(z) d z \in W$ and $g(y)-g(x)=\int_{x}^{y} f(z) d z \in W$.

Let $t \in E$ and $r \in R$ such that $x+r t, y+r t \in U$. Then

$$
(y+r t)-(x+r t)=y-x \in S,
$$

and hence $g(y+r t)-g(x+r t) \in W$. Thus for all $t \in E$

$$
\begin{aligned}
f(y)(t)-f(x)(t) & =\lim _{r \rightarrow 0}[g(y+r t)-g(y)] r^{-1}-\lim _{r \rightarrow 0}[g(x+r t)-g(x)] r^{-1} \\
& =\lim _{r \rightarrow 0}\{[g(y+r t)-g(x+r t)]-[g(y)-g(x)]\} r^{-1} \in W .
\end{aligned}
$$


5. Strong maximum modulus theorem and the uniqueness theorem. The principle result of this section is $(A)$. The three lemmas of this section involving orientation preserving operators are also used to obtain $(B)$ in $\S 6$. A simple consequence of $(A)$ or $(B)$ is the uniqueness theorem which states that an integrable function of the family of functions in question, defined on $\bar{U}$, is uniquely determined by its value on the boundary $V$ of $\bar{U}$. Indeed by the same methods used to prove $(A)$, the uniqueness theorem can be shown to hold for an arbitrary algebra of integrable functions, defined on $\bar{U}$, without any condition of commutivity being placed on the algebra.

(A) makes no requirement that the family of functions in question even form on algebra. All results in this paper with the exception of $(A)$ and the uniqueness theorem are stated only for the commutative case. It is conjectured that some kind of maximum modulus theorem holds for noncommutative algebras of integrable functions.

From the standpoint of [3] the most obvious example of an orientation preserving family is $Z=\{A \in B ; A I=I A\}$, where $I$ is an element of $B$ such that $I^{2}=-e$. In this case one can interpret $E$ as a complex Euclidean space and $Z$ as the family of complex homogeneous linear operators acting on $E$. A less obvious but important example is $Z_{0}=\left\{A \in B ; A^{k}=0\right.$ for some $\left.k \in \omega\right\}$.

Let $r \in R, r>0, A \in Z_{0}$, and suppose $A+r e$ does not invert. Then there exists $x \in E, x \neq 0$, such that $(A+r e)(x)=0$. Then for some $k \in \omega, A^{k}=0, A(x)=-r x$, and $0=0(x)=A^{k}(x)=(-1)^{k} r^{k} x$, and $x=0$. Thus $A+r e$ inverts for all $r>0$, and hence from Lemma 5.3, below, $A+r e$ is orientation preserving for all $r>0$. In $\$ 7$ it shall be shown for $A \in B$, that $A \in Z_{0}$ if and only if $\|A\|_{s}=0$. Let $F_{0}$ be the family of all integrable functions on open subsets of $E$ into $Z_{0}$.

A simple example of an orientation preserving family that is a commutative algebra is that generated by the operator $L$ acting on $E_{n}=R \oplus R \oplus R \oplus \cdots \oplus R, n \in \omega$, such that for $\left(x_{1}, \cdots, x_{n}\right) \in E_{n}$, $L\left(x_{1}, \cdots, x_{n}\right)=\left(x_{2}, \cdots, x_{n}, 0\right)$. Jlearly $L^{n}=0$.

The following two lemmas show that for a suitably chosen family of integrable functions, the integrals of the elements of $F$ satisfy a maximum modulus theorem, allowing us to obtain a maximum modulus theorem $(A)$ for the elements of $F$ proper.

LEMmA 5.1. Let $f$ be a (Frechét) differentiable function on an open set in $E$ into $E$ such that:

(1) $\bar{U} \subseteq$ domain $f$, and for $x \in U$, if $f_{x}^{\prime}$ is an invertible element of $B$, then $f_{x}^{\prime}$ is orientation preserving. 
(2) There exists a dense subset $H$ of $U$ such that for $x \in H, f_{x}^{\prime}$ is invertible.

Then for $x \in U,\|f(x)\| \leqq \sup \{\|f(t)\| ; t \in V\}$.

Proof. This lemma may be found in [3]. The proof employs degree theoretic methods from algebraic topology.

LEMMA 5.2. Let $\rho>1$, and $f$ an integrable function on $U(\rho)$ into $B$ such that for $x \in U(p), r>0$, if $f(x)+r e$ inverts, then $f(x)+$ re is orientation preserving. Let $F^{\prime}$ be an algebra (not necessarily commutative) of integrable functions on $U(\rho)$ into $B$, with identity, and let $g \in F^{\prime}$. Set $u(x)=\int_{0}^{x} f(z) d z$ and $v(x)=\int_{0}^{x} g^{2}(z) d z$ for $x \in U(\rho)$. Then for $w=u, v, x \in U$,

$$
\|w(x)\| \leqq M=\sup \{\|f(t)\| ; t \in V\} .
$$

Proof. Set $w=u$ or $v$, and $H_{w}=\left\{x \in U\right.$; $w_{x}^{\prime}$ inverts $\}$. If $H_{w}$ is dense in $U$, the lemma follows from Lemma 5.1. Suppose now that $H_{w}$ is not dense in $U$. We shall prove the lemma by uniformly approximating $w$ by functions satisfying the hypothesis of Lemma 5.1.

Let $x_{1}, x_{2}, \cdots$ be a countable dense subset of $U$. For $i \in \omega$, set $C_{i}=\left\{r \in R ; f\left(x_{i}\right)+r e\right.$ or $g\left(x_{i}\right)+r e$ does not invert $\}$. Since $E$ is finite dimensional, $C_{i}$ must be finite for $i \in \omega$, and thus $C=U_{1}^{\infty} C_{i}$ must be countable. Let $r_{1}>r_{2}>\cdots$ be a sequence in $R-C$ which converges to 0 , and for $x \in U(\rho), i \in \omega$, set $u_{i}(x)=\int_{0}^{x}\left[f(z)+r_{i} z\right] d z$ and $v_{i}(x)=\int_{0}^{x}\left[g(z)+r_{i} z\right]^{2} d z$.

Let $i \in \omega, x \in U(\rho)$. Then $\left(u_{\imath}\right)_{x}^{\prime}=f(x)+r_{i} e$ is orientation preserving if invertible by hypothesis, and trivially $\left[g(x)+r_{i} e\right]^{2}$ is orientation preserving if invertible. For $i, j \in \omega$, since $r_{i} \in C, r_{i} \notin C_{j}$, and hence $f\left(x_{j}\right)+r_{i} e$ and $g\left(x_{j}\right)+r_{i} e$ invert. Whence $\left[g\left(x_{j}\right)+r_{i} e\right]^{2}$ inverts. Then from Lemma 5.1, for $i \in \omega, x \in U, w_{\imath}=u_{i}$ or $v_{i}$,

$$
\left\|w_{i}(x)\right\| \leqq \sup \left\{\left\|w_{\imath}(t)\right\| ; t \in V\right\} \text {. }
$$

Letting $i \rightarrow 0, w_{i} \rightarrow w$, and the lemma follows.

Theorem 5.1. (Strong maximum modulus theorem). Let $\rho>1$, and let $f$ be an integrable function on $U(\rho)$ into an orientation preserving family $G$ of $B, G$ a linear subspace of $B$. Then for $x \in U$,

$$
\|f(x)\| \leqq M=\sup \{\|f(t)\| ; t \in V\} \text {. }
$$

Proof. Let $F^{\prime}$ bə the family of all integrable functions on open 
subsets of $E$ into $G$. Since $G$ is a linear space, $F^{\prime}$ is a linear space closed under the operation of translation.

For $x \in U(\rho)$, set $u(x)=\int_{0}^{x} f(z) d z$. For $\alpha \in V, 0<r<(\rho-1) / 2$, $x \in U[(1+\rho) / 2]$, set $u(\alpha, r)(x)=[u(x+r \alpha)-u(x)] r^{-1}$. Let $\varepsilon>0$. Then there exists $0<\delta<\rho-1$, such that for $x \in E, 1-\delta \leqq\|x\| \leqq$ $1+\hat{o}$, we have $\|f(x)\| \leqq M+\varepsilon$. Then for $0<r<\hat{o}, x, \alpha \in V$,

$$
\begin{aligned}
\|u(r, \alpha)(x)\| & =\left\|[u(x+r \alpha)-u(x)] r^{-1}\right\| \\
& \left.=\left\|\int_{x}^{x+r \alpha} f(z) d z\right\| r^{-1} \leqq(M+\varepsilon) \|(x+r \alpha)-x\right] \| r^{-1} \\
& =(M+\varepsilon)\|r \alpha\| r^{-1} \\
& =M+\varepsilon .
\end{aligned}
$$

For $x \in U, 0<r<\delta, \alpha \in V$, we have

$$
u(r, \alpha)_{s}^{\prime}=[f(x+r \alpha)-f(x)] r^{-1} \in G,
$$

and hence from Lemma 5.2 ,

$$
\|u(r, \alpha)(x)\| \leqq \sup \{\|u(r, \alpha)(t)\| ; t \in V\} \leqq M+\varepsilon,
$$

and

$$
\text { \|f } f(x)(\alpha)\left\|=\lim _{r \rightarrow 0}\right\| u(r, \alpha)(x) \| \leqq M+\varepsilon .
$$

Thus for $x \in U, \alpha \in V$, since $\varepsilon$ is arbitrary, $\|f(x)(\alpha)\| \leqq M$, and thus \|f $f(x) \| \leqq M$.

One additional lemma and we will be able to obtain the uniqueness theorem.

Lemma 5.3. Let $A \in T$. Then:

(1) If $p$ is even and there exists at most one element $r \in R$ such that $A+r e$ does not invert, then if $A$ inverts, $A$ is orientation preserving.

(2) If $p$ is odd, there exists at least one element $r \in R$ such that $A+$ re does not invert.

(3) If for all $r \in R, r \neq 0, A+$ re inverts, then $A+$ re is orientation preserving for all $r>0$.

Proof. Let $\alpha \in\{-1,+1\}$ and suppose for all $s \geqq 0$, that $A+\alpha s$ is invertible. Now extend the reals to include $-\infty$ and $+\infty$ and set $f(s)=A+\alpha s e$ for $0 \leqq s \leqq 1$, set $f(s)=A / s+\alpha e$ for $1<s<\infty$ and set $f(\infty)=\alpha e$. Then $f$ is a continuous map of $[0, \infty]$ into the space of invertible elements of $B$ and hence

$$
\mu(A)=\mu[f(0)]=\mu[f(\infty)]=\mu(\alpha e) .
$$


We now handle (1). For some $\alpha \in\{-1,+1\}, A+s \alpha e$ inverts for all $s \geqq 0$. Thus $\mu(A)=\mu(\alpha e)$. Since $p$ is even $\mu(-e)=\mu(+e)=1$, and thus $u(A)=1$.

We now handle (2). If $A+s e$ inverts for all $s \in R$, we have, since $p$ is odd, $1=\mu(e)=\mu(A)=\mu(-e)=-1$.

We now handle (3). Let $r>0$ and set $A_{0}=A+r e$. Then $A_{0}+s e$ inverts for all $s \geqq 0$ and hence $\mu(A+r e)=\mu\left(A_{0}\right)=\mu(e)=1$.

To prove the lemma employing determinants, we observe that if we set $h(s)=\operatorname{det}(A+s e)$, then $h$ is a polynomial of degree $p$ which vanishes if and only if $A+s e$ does not invert. Moreover for $s \in R$, if $h(s)>0$, then $\mu(A+s e)=1$ and if $h(s)<0, \mu(A+s e)=-1$. The lemma would then follow from these facts:

(1) If $p$ were even and $h$ had at most one root $r_{0}$, then $h(s)>0$ for all $s \in R, s \neq r_{0}$.

(2) If $p$ were odd, then $\lim _{s \rightarrow-\infty} h(s)=-\infty$ and $\lim _{s \rightarrow \infty} h(s)=\infty$, and thus for some $r_{0} \in R, h\left(r_{0}\right)=0$.

(3) $h(s) \neq 0$ for $s \in(0, \infty)$ and $\lim _{s \rightarrow \infty} h(s)=\infty$, implies $h(s)>0$ for all $s \in(0, \infty)$.

Theorem 5.2. (uniqueness theorem). Let $\rho>1$, and let $F^{\prime}$ be an algebra (not necessarily commutative) of integrable functions on $U(\rho)$ into $B$, with identity, and let $f, g \in F^{\prime}$. Then if $f(t)=g(t)$ for all $t \in V$, then $f(x)=g(x)$ for all $x \in U$.

Proof. Set $h=f-g$, let $x_{0} \in V$, and set $u(x)=\int_{x_{0}}^{x} h^{2}(z) d z$ for $x \in U(\rho)$. Then for all $x \in V, h^{2}(x)=h(x)=0$, and thus for all $x \in V$, $u(x)=0$. Hence from Lemma 5.2, for all $x \in U$,

$$
\|u(x)\| \leqq \sup \{\|u(t)\| ; t \in V\}=0,
$$

and $u(x)=0$. Hence for $x \in U, t \in E$,

$$
h^{2}(x)(t)=\lim _{r \rightarrow 0}[u(x+r t)-u(x)] r^{-1}=\lim _{r \rightarrow 0} 0 / r=0
$$

and $h^{2}(x)=0$.

Thus range $h$ lies in $Z_{0}$ and $h \in F_{0}$. For $x \in U(\rho)$, set

$$
v(x)=\int_{x_{0}}^{x} h(z) d z .
$$

Then $v(x)=0$ for $x \in V$. From Lemma 5.3, $Z_{0}$ is an orientation preserving family. Hence we may apply Lemma 5.2 to $v$ and obtain as above in the case of $u$, for $x \in U,(f-g)(x)=h(x)=0$ and $f(x)=g(x)$.

6. The weak maximum modulus theorem. 
TheoRem 6.1. There exists $N>0$, such that for $\delta>1, f \in F$, domain $f=U(\delta)$, we have for $x \in U$,

$$
\|f(x)\| \leqq N M,
$$

where $M=\sup \{\|f(t)\| ; t \in V\}$.

Proof. For $x \in U(\delta)$, set $g(x)=\int_{0}^{x} f(z) d z$. We consider first the case when there exist two distinct irreducible invariant subspaces $H_{1}, H_{2}$ of $E$. In this case we shall employ $H_{1}$ ond $H_{2}$ to form a new norm on $T,\|\cdot\|_{0}$, and maximize with respect to $\|\cdot\|_{0}$.

For $i=1,2$, let $\theta_{i}$ be the natural homomorphism of $E$ onto $E_{i}=$ $E / H_{i}$. For $i=1,2, A \in T$, set $\|A\|_{i}=\sup \left\{\left\|\theta_{i} A(t)\right\| ; t \in V\right\}$, and set $\|A\|_{0}=\|A\|_{1}+\|A\|_{2}$. Suppose $A$ is an element of $E$ such that $\|A\|_{0}=0$. If we show that $A=0$, we will have that $\|\cdot\|_{0}$ is a norm on $T$.

Let $x \in E$. Now for $i=1,2,\left\|\theta_{i} A\right\|=0$ and $\theta_{i} A=0$, and hence $0=\left[\theta_{i} A\right](x)=\theta_{i}[A(x)]$. Thus $A(x) \in H_{i}$ for $i=1,2$, and thus $A(x)$ lies in the invariant subspace $H_{1} \cap H_{2}$ of $E$. From the minimality of $H_{1}, H_{2}, H_{1} \cap H_{2}=\{0\}$, and thus $A(x)=0$. Thus $A=0$ and $\|\cdot\|_{0}$ is a norm.

Let $i=1,2$, and let $x, y \in U$ such that $y-x \in H_{i}$. Then setting $S=W=H_{i}$, we have from Lemma 4.2, for all $t \in E,[f(y)-f(x)](t) \in H_{i}$, and hence $\theta_{i} f(y)(t)-\theta_{i} f(x)(t)=\theta_{i}\{[f(y)-f(x)](t)\}=0$. Thus $\theta_{i} f(y)=$ $\theta_{i} f(x)$ and $\|f(y)\|_{i}=\|f(x)\|_{i}$.

Now there exist $0<\alpha<\beta$ such that for $A \in T$,

$$
\alpha\|A\| \leqq\|A\|_{0} \leqq \beta\|A\| \text {. }
$$

Let $x \in U$. Then there exist $y_{1}, y_{2} \in V$, such that $y_{i}-x \in H_{i}$ for $i=$ 1,2 , and thus

$$
\begin{aligned}
\alpha\|f(x)\| & \leqq\|f(x)\|_{0}=\|f(x)\|_{1}+\|f(x)\|_{2}=\left\|f\left(y_{1}\right)\right\|_{1}+\left\|f\left(y_{2}\right)\right\|_{2} \\
& \leqq\left\|f\left(y_{1}\right)\right\|_{0}+\left\|f\left(y_{2}\right)\right\|_{0} \leqq \beta\left\|f\left(y_{1}\right)\right\|+\beta\left\|f\left(y_{2}\right)\right\| \leqq 2 \beta M,
\end{aligned}
$$

and thus $\|f(x)\| \leqq N M$, where $N=2 \beta \alpha^{-1}$.

We now consider the case where there exists exactly one irreducible invariant subspace $H$ of $E$. If the dimension $p$ of $E$ is even we will show that the hypothesis of Theorem 5.1 is satisfied. If $p$ is odd, we will be able, if $T$ does not possess nonzero elements with ranges lying in $H$, to reduce the argument to that employed in the case of two invariant subspaces. Otherwise we shall be forced to engage in a lengthy and detailed structural analysis of $T$ and $f$.

Let $A \in T$. Then there exists at most one element $r \in R$ such that $A-r e$ does not invert. Indeed suppose that there exist 
$r_{1}, r_{2} \in R, r_{1} \neq r_{2}$ such that $A-r_{1} e$ and $A-r_{2} e$ do not invert. Let $i=1,2$, and set $V_{i}=\left\{x \in E ;\left(a-r_{i} e\right)(x)=0\right\}$. Then for $x \in V_{i}, B \in T$, $y=B(x)$, we have

$$
\left(A-r_{i} e\right)(y)=\left[\left(A-r_{i} e\right) B\right](x)=B\left[\left(A-r_{i} e\right)(x)\right]=B(0)=0,
$$

and thus $V_{i}$ is an invariant subspace of $E$. Now $V_{i}$ must contain an irreducible invariant subspace $P_{i}$, and since $H$ is unique, $P_{i}=H$. Thus $P_{1}=P_{2}=H$, and for $x \in H, x \neq 0,0=A(x)-A(x)=r_{1} x-r_{2} x=$ $\left(r_{1}-r_{2}\right) x$. But then $r_{1} \neq r_{2}$ implies $x=0$.

If $p$ is even, then from Lemma 5.3, $T$ is an orientation preserving family, and hence from Theorem 5.1, (1) holds with $N=1$.

We now consider the case when $p$ is odd. Suppose for $\sigma \in T$, $\sigma(E) \leqq H$, implies $\sigma=0$, let $\theta$ be the natural homomorphism of $E$ into $E / H$, and set $\|A\|^{\prime}=\|\theta A\|$ for $A \in T$. Then $\|\cdot\|^{\prime}$ is a norm on $T$ and the argument reduces to that for $\|\cdot\|_{0}$.

Now suppose there exists $\sigma_{0} \in T, \sigma_{0} \neq 0$, such that $\sigma_{0}(E) \subseteq H$. We shall write $E$ as the direct sum of the null space $S$ of $\sigma_{0}$ and a one-dimensional subspace $W$ of $E$, and shall write $f$ in the form $\rho e+\sigma$, where $\sigma(x)(y)=0$ for $x \in U(\rho), y \in H$. We will show that $\rho$ is constant on $S$ and thus that $\sigma$ is integrable on $S$ (and hence on all hyperplanes parallel to $S$ ) and that since $S$ has even dimension $p-1$, that $\sigma \mid S$ satisfies (1). It then remains to relate the behavior of $\sigma$ in the direction of $W$ to $\rho$ to complete the argument.

We decompose $f$. Since $p$ is odd, from Lemma 5.3, for $A \in T$, there exists $\rho_{A} \in R$ such that $\sigma_{A}=A-\rho_{A} e$ does not invert. For $x \in U(\rho), A=f(x)$, set $\rho(x)=\rho_{A}$ and set $\sigma(x)=\sigma_{A}$. Then $f=\rho e+\sigma$.

We decompose $E$. Set $S=\left\{x \in E ; \sigma_{0}(x)=0\right\}$, let $x_{0} \in V$ such that $\sigma_{0}\left(x_{0}\right) \neq 0$, and set $W=\left\{r x_{0} ; r \in R\right\}$. If $H$ is shown to be one-dimensional, then since $\sigma_{0}(E) \subseteq H$, we would have that range $\sigma_{0}$ is onedimensional, and thus that the null space $S$ of $\sigma_{0}$ is $p-1$ dimensional, giving us $E=S \oplus W$. Let $\beta \in H, \quad \beta \neq 0$. Now for all $A \in T$, $H \subseteq\left\{x \in E ;\left[A-\rho_{A} e\right](x)=0\right\}$, and thus $\sigma_{A}(\beta)=\left[A-\rho_{A}\right](\beta)=0$ and $A(\beta)=\rho_{A} \beta$. Then $H^{\prime}=\{r \beta ; r \in R\}$ is an invariant subspace of $E$ lying in $H$, and thus $H^{\prime}=H$ and $H$ is one-dimensional. Also $\sigma(x)(H)=\{0\}$ for $x \in U(\rho)$.

We now show that $\rho$ is constant on $S$. Consider $\rho \sigma_{0}=f \sigma_{0}-\sigma \sigma_{0}$. We first show that $\sigma \sigma_{0} \equiv 0$ and thus that $\rho \sigma_{0}=f \sigma_{0}$, and therefore that $\rho \sigma_{0}$ is integrable.

Let $x \in U(\rho)$ and set $\alpha=\sigma(x)$. Then $\alpha(\beta)=0$. Now

$$
E \supseteqq \alpha(E) \supseteqq \alpha^{2}(E) \supseteqq \ldots
$$

is a sequence of invariant subspaces of $E$, and $H$ must lie in $\alpha^{k}(E)$, if $\alpha^{k}(E) \neq(0)$ for $k \in \omega$. Suppose $\alpha^{p} \neq 0$. Then $\alpha^{p}(E) \neq\{0\}$ and for 
some $1 \leqq k<p, \quad k \in \omega, \quad \alpha^{k}(E)=\alpha^{k+1}(E)=\alpha\left[\alpha^{k}(E)\right] \neq\{0\}$ and $\alpha$ is one-to-one on $\alpha^{k}(E)$. But then $\beta \in H \subseteq \alpha^{k}(E)$ and $\alpha(\beta) \neq 0$. Thus $\alpha^{p}=0$. Now for some $x_{1} \in S, s \in R, \alpha\left(x_{0}\right)=x_{1}+s x_{0}$. Then $\alpha^{2}\left(x_{0}\right)=$ $\alpha\left(x_{1}\right)+s \alpha\left(x_{0}\right)=\alpha\left(x_{1}\right)+s\left[x_{1}+s x_{0}\right]=x_{2}+s^{2} x_{0}$, where $x_{2}=\alpha\left(x_{1}\right)+s x_{1} \in S$. Continuing, we obtain $0=\alpha^{p}\left(x_{0}\right)=x_{p}+s^{p} x_{0}$, where $x_{p} \in S$ and $s^{p} x_{0} \in W$. Thus $x_{p}=s^{p} x_{0}=0$ and $s^{p}=0, s=0$, and $\alpha\left(x_{0}\right)=x_{1} \in S$. Thus $\alpha(E) \subseteq S$. Now $\sigma_{0}(S)=\{0\}$, and thus $\alpha \sigma_{0}(E)=\sigma_{0}[\alpha(E)] \subseteq \sigma_{0}(S)=$ $\{0\}$ and $\alpha \sigma_{0}=0$.

For $z \in U, t \in S$, we have $\left[\rho(z) \sigma_{0}\right](t)=0$. Therefore, setting $W=$ $\{0\}$, we have from Lemma 4.2 , for $x, y \in E$ such that $y-x \in S$, that $\left[\rho(y) \sigma_{0}-\rho(x) \sigma_{0}\right](t)=0$ for all $t \in E$. In particular

$$
0=\left[\rho(y) \sigma_{0}-\rho(x) \sigma_{0}\right]\left(x_{0}\right)=[\rho(y)-\rho(x)] \sigma_{0}\left(x_{0}\right)
$$

and hence since $\sigma_{0}\left(x_{0}\right) \neq 0$, we have $\rho(y)-\rho(x)=0$ and $\rho(y)=\rho(x)$. Thus on hyperplanes of the form $x+S, x \in E, \rho$ is constant and $\sigma=f-\rho e$ is integrable. For $s \in[-1,1]$, set $\phi(s)=\rho\left(s x_{0}\right)$.

We now study the relationship between $\sigma(\cdot)\left(x_{0}\right)$, and $\rho$. Let $s_{0} \in R,\left|s_{0}\right|<1$, and let $x, y$ be distinct points of the line $L=$ $\left\{s_{0} x_{0}+s \beta ; s \in R\right\}$ such that $x, y \in \bar{U}$. Then $\rho(y)=\rho(x)=\phi\left(s_{0}\right)$. Since $S$ is an invariant subspace of $E, \beta \in H \cong S$, and thus $y-x \in S$. Now there exists $\pi_{0}>0$ such that for $0<\pi<\pi_{0}$ the contour $C=$ $[x, y] \cup\left[y, y+\pi x_{0}\right] \cup\left[y+\pi x_{0}, x+\pi x_{0}\right] \cup\left[x+\pi x_{0}, x\right]$ as well as its interior lies in $U(\delta)$.

Let $0<\pi<\pi_{0}$. Since $\sigma(z)(\beta)=0$ for all $z \in U(\delta)$,

$$
\int_{x}^{y} \sigma(z) d z=0 \quad \text { and } \quad \int_{x+\pi x_{0}}^{y+\pi x_{0}} \sigma(z) d z=0 .
$$

Moreover, since for $0 \leqq s \leqq \pi,\left(x+s x_{0}\right)-\left(y+s x_{0}\right)=x-y \in S$, we have $\rho\left(x+s x_{0}\right)=\rho\left(y+s x_{0}\right)$ and thus

$$
\int_{x}^{x+\pi x_{0}} \rho(z) d z=\int_{y}^{y+\pi x_{0}} \rho(z) d z
$$

Thus

$$
\begin{aligned}
0= & \int_{C} f(z) d z=\int_{C} \sigma(z) d z+\int_{C} \rho(z) e d z \\
= & {\left[\int_{x}^{x+\pi x_{0}} \sigma(z) d z-\int_{y}^{y+\pi x_{0}} \sigma(z) d z\right] } \\
& +\rho(x)(y-x)-\rho\left(x+\pi x_{0}\right)\left[\left(y+\pi x_{0}\right)-\left(x+\pi x_{0}\right)\right] .
\end{aligned}
$$

Hence since $\rho(x)=\dot{\phi}\left(s_{0}\right)$ and $\rho\left(x+\pi x_{0}\right)=\phi\left(s_{0}+\pi\right)$, we have

$$
\left[\phi\left(s_{0}+\pi\right)-\phi\left(s_{0}\right)\right] \pi^{-1}(y-x)=\pi^{-1} \int_{x}^{x+\pi x_{0}} \sigma(z) d z-\pi^{-1} \int_{y}^{y+\pi x_{0}} \sigma(z) d z
$$

Now as $\pi \rightarrow 0$, the right hand side of (2) must converge to 


$$
\sigma(x)\left(x_{0}\right)-\sigma(y)\left(x_{0}\right)
$$

and thus $\phi^{\prime}\left(s_{0}\right)$ exists and

$$
\dot{\phi}^{\prime}\left(s_{0}\right)(y-x)=\sigma(x)\left(x_{0}\right)-\sigma(y)\left(x_{0}\right) .
$$

Let $x^{\prime}, y^{\prime} \in L \cap V, x^{\prime} \neq y^{\prime}$. Then $\dot{\phi}^{\prime}\left(s_{0}\right)\left(y^{\prime}-x^{\prime}\right)=\sigma\left(x^{\prime}\right)\left(x_{0}\right)-\sigma\left(y^{\prime}\right)\left(x_{0}\right)$, and

$$
\left|\phi^{\prime}\left(s_{0}\right)\right|=\left\|\sigma\left(x^{\prime}\right)\left(x_{0}\right)-\sigma\left(y^{\prime}\right)\left(x_{0}\right)\right\| \cdot\left\|y^{\prime}-x^{\prime}\right\|^{-1} \leqq 2 M_{\sigma}\left\|y^{\prime}-x^{\prime}\right\|^{-1},
$$

where $M_{\sigma}=\sup \{\|\sigma(t)\| ; t \in V\}$. Then for $x \in L \cap U, \phi^{\prime}\left(s_{0}\right)\left(x-x^{\prime}\right)=$ $\sigma(x)\left(x_{0}\right)-\sigma\left(x^{\prime}\right)\left(x_{0}\right)$, and

$$
\begin{aligned}
\left\|\sigma(x)\left(x_{0}\right)\right\| & =\left\|\phi^{\prime}\left(s_{0}\right)\left(x-x^{\prime}\right)+\sigma\left(x^{\prime}\right)\left(x_{0}\right)\right\| \\
& \leqq\left\|\phi^{\prime}\left(s_{0}\right)\right\| \cdot\left\|x-x^{\prime}\right\|+\left\|\sigma\left(x^{\prime}\right)\left(x_{0}\right)\right\| \\
& \leqq 2 M_{\sigma}\left\|y^{\prime}-x^{\prime}\right\|^{-1}\left\|x-x^{\prime}\right\|+M_{\sigma} \\
& \leqq 3 M_{\sigma}
\end{aligned}
$$

where clearly $\left\|y^{\prime}-x^{\prime}\right\|^{-1}\left\|x-x^{\prime}\right\| \leqq 1$.

We are now ready for the concluding arguments. For $\alpha \in T$, set $\|\alpha\|_{1}=\sup \{\|\alpha(t)\| ; t \in S \cap V\}$ and set $\|\alpha\|_{2}=\|\alpha\|_{1}+\left\|\alpha\left(x_{0}\right)\right\|$. Since $S$ has even dimension $p-1$, we have for $x \in U$ and the hyperplane $S_{x}=x+S$,

$$
\|\sigma(x)\|_{1} \leqq \sup \left\{\|\sigma(t)\|_{1} ; t \in S_{x} \cap V\right\} \leqq M_{\sigma}
$$

Since $T$ is the direct sum of $\{r e ; r \in R)$ and $D=\left\{\alpha \in T ; \alpha^{p}=0\right\}$ the mappings

$$
P: r e+\alpha \longrightarrow r \text { and } Q: r e+\alpha \longrightarrow \alpha \quad(r \in R ; c: \in D)
$$

are uniquely defined linear transformations. Hence there exists $N_{0}>0$ such that for $z=r e+\alpha, r \in R, \alpha \in D$,

$$
|r|=|P(z)| \leqq N_{0}\|z\| \text { and }\|\alpha\|=\|Q(z)\| \leqq N_{0}\|z\|,
$$

and thus

$$
M_{\sigma} \leqq N_{0} M
$$

Clearly $\|\cdot\|_{2}$ is a norm on $T$ and hence there exists $N_{1}>0$ such that $\|\alpha\| \leqq N_{1}\|\alpha\|_{2}$ for $\alpha \in T$. Then from equations (3)-(6) for $x \in U$, we have for some $y \in V$,

$$
\begin{aligned}
\|f(x)\| & =\|\rho(x) e+\sigma(x)\| \leqq\|\rho(x) e\|+\|\sigma(x)\| \leqq|\rho(x)|+N_{1}\|\sigma(x)\|_{2} \\
& =|\rho(y)|+N_{1}\|\sigma(x)\|_{1}+N_{1}\left\|\sigma(x)\left(x_{0}\right)\right\| \\
& \leqq N_{0} M+N_{1} M_{\sigma}+N_{1}\left(3 M_{\sigma}\right) \\
& \leqq N_{0} M+N_{1} N_{0} M+3 N_{1} N_{0} M=N M,
\end{aligned}
$$


where $N=N_{0}\left(1+4 N_{1}\right)$.

7. Maximal spectral norm theorem. Let $f \in F, \bar{U} \cong$ domain $f$, $x \in U$. In this section we show that

$$
\|f(x)\|_{s} \leqq \sup \left\{\|f(t)\|_{s} ; t \in V\right\} .
$$

Now for $n \in \omega, f^{n} \in F$, and from the weak maximum modulus theorem, Theorem 6.1, there exists $N>0$, determined by $F$, such that

$$
\left.\left\|f^{n}(x)\right\| \leqq N \sup \left\{\left\|f^{n}(t)\right\|\right\} ; t \in V\right\},
$$

and hence

$$
\left\|f(x)^{n}\right\|^{1 / n} \leqq N^{1 / n} \sup \left\{\left\|f(t)^{n}\right\|^{1 / n} ; t \in V\right\} \text {. }
$$

If we let $n \rightarrow \infty$, we obtain $\|f(x)\|_{s}$ from the left hand side of (1), and would expect to obtain $\sup \left\{\|f(t)\|_{s} ; t \in V\right\}$ from the right hand side of (1), thus yielding us $(C)$. The latter conclusion, however, is not obvious and requires Lemma 7.3 in its proof. Thus, especially in view of the lengthy and highly technical proof of Theorem 6.1, it is highly desirable to have a direct proof of $(C)$; which is given in Theorem 7.2.

To this end we first prove $(C)$ for a semi-norm $\|\cdot\|_{m}$ defined in terms of the family $\mathscr{C}$ of irreducible subspaces of $E$ invariant under $T$ and show that this new semi-norm coincides with the spectral semi-norm.

For $H \in \mathscr{C}, x \in T$, setting $x_{0}=x \mid H$, set

$$
\|x\|_{H}=\left\|x_{0}\right\|_{s}=\lim \sup _{n \rightarrow \infty}\left\|x_{0}^{n}\right\|^{1 / n} .
$$

Then for $x \in T$, we set $\|x\|_{m}=\sup \left\{\|x\|_{I I} ; H \in \mathscr{C}\right\}$. Clearly $\|x\|_{m} \leqq$ $\|x\|_{s} \leqq\|x\|$ for all $x \in T$.

Let $H \in \mathscr{X}$ and set $Z=T \mid H$. From Lemma $4.1, Z$ is a field, and from the fundamental theorem of algebra, $Z$ is isomorphic to $R$ or $K$. However, the spectral norm need not coincide with the natural operator norm on $Z$. Hence $Z$ under the natural operator norm need not be isometric to $R$ or $K$.

Let $f$ be a functional on $T . \quad f$ is said to be a semi-norm if for all $x, y \in T, r>0$, we have

(1) $f(x) \geqq 0$.

(2) $f(r x)=r f(x)$.

(3) $f(x+y) \leqq f(x)+f(y)$.

(4) $f(x y) \leqq f(x) f(y)$.

(5) $f(e)=1$.

$f$ is said to be multiplicative if $f\left(x^{2}\right)=f(x)^{2}$ for $x \in T$. 
We now show that the spectral norm is a multiplicative semi-norm and hence trivially that $\|\cdot\|_{m}$ is a multiplicative semi-norm. This can be obtained by complexifying $T$ and applying standard arguments. A direct argument for the real case consonant with the real variable approach of this paper is given by Riesz-Nagy [7] and is quite simple.

THEOREM 7.1. For $x \in T$, set $f(x)=\|x\|_{s}=\lim \sup _{n \rightarrow \infty}\left\|x^{n}\right\|^{1 / n}$. Then $f$ is a multiplicative semi-norm on $T$.

Proof. (Riesz-Nagy) Let $x \in T$. We first show that $\lim _{n \rightarrow \infty}\left\|x^{n}\right\|^{1 / n}$ exists. Setting $a_{n}=\left\|x^{n}\right\|$ for $n \in \omega$, we have for $n, m \in \omega, a_{n+m}=$ $\left\|x^{n+m}\right\| \leqq\left\|x^{n}\right\| \cdot\left\|x^{m}\right\|=a_{n} a_{m}$. Set $\alpha=\inf \left\{a_{n}^{1 / n} ; n \in \omega\right\}$ and let $\varepsilon>0$. Then for some $m \in \omega, a_{m}^{1 / m}<\alpha+\varepsilon / 2$. Let $n>m, n \in \omega$. Then $n=$ $m q+r$ where $q \in \omega$, and $0 \leqq r<m$. Clearly $a_{n} \leqq\left(a_{m}\right)^{q} a_{r}$ and thus $a_{n}^{1 / n} \leqq a_{n}^{q / n} a_{r}^{1 / n}=\left(a_{m}^{1 / n}\right)^{q m / n} a_{r}^{1 / n} \leqq(\alpha+\varepsilon / 2)^{q m / n} a_{r}^{1 / n}$. Now as $n \rightarrow \infty$, we have $q m / n=q m /(q m+r) \rightarrow 1$ and $a_{r}^{1 / n} \rightarrow 1$, and thus for $n$ sufficiently large $\alpha \leqq a_{n}^{1 / n} \leqq \alpha+\varepsilon$. Thus $\lim _{n \rightarrow \infty}\left\|x^{n}\right\|^{1 / n}$ exists and is equal to $\alpha$.

Now for $x, y \in T$,

$$
\begin{aligned}
\|x y\|_{s} & =\lim _{n \rightarrow \infty}\left\|(x y)^{n}\right\|^{1 / n} \\
& =\lim _{n \rightarrow \infty}\left\|x^{n} y^{n}\right\|^{1 / n} \leqq \lim _{n \rightarrow \infty}\left\|x^{n}\right\|^{1 / n}\left\|y^{n}\right\|^{1 / n} \\
& =\|x\|_{s}\|y\|_{s},
\end{aligned}
$$

and

$$
\left\|x^{2}\right\|_{s}=\lim _{n \rightarrow \infty}\left\|\left(x^{2}\right)^{n}\right\|^{1 / n}=\left[\lim _{n \rightarrow \infty}\left\|x^{2 n}\right\|^{1 / 2 n}\right]^{2}=\|x\|_{s}^{2} .
$$

Let $A, B \in T$. We now show that $\|A+B\|_{s} \leqq\|A\|_{s}+\|B\|_{s}$. Set $a=\|A\|$ and $b=\|B\|$ and let $\varepsilon>0$. Then for some $m \in \omega$, $\left\|A^{n}\right\|^{1 / n}<p=\|A\|_{s}+\varepsilon / 2$ and $\left\|B^{n}\right\|^{1 / n}<q=\|B\|_{s}+\varepsilon / 2$ for all $n \geqq$ $m, n \in \omega$. Thus for $n \geqq 2 m, n \in \omega$,

$$
\begin{aligned}
& \left\|(A+B)^{n}\right\| \leqq \sum_{i=0}^{m-1}\left(\begin{array}{c}
n \\
i
\end{array}\right) a^{i} q^{n-i}+\sum_{i=m}^{n-m}\left(\begin{array}{c}
n \\
i
\end{array}\right) p^{i} q^{n-i}+\sum_{i=n-m+1}^{n}\left(\begin{array}{c}
n \\
i
\end{array}\right) p^{i} b^{n-i} \\
= & \sum_{i=0}^{m-1}\left(\begin{array}{c}
n \\
i
\end{array}\right) p^{i} q^{n-i}(a / p)^{i}+\sum_{i=m}^{n-m}\left(\begin{array}{c}
n \\
i
\end{array}\right) p^{i} q^{n-i}+\sum_{i=n-m+1}^{n}\left(\begin{array}{c}
n \\
i
\end{array}\right) p^{i} q^{n-i}(b / q)^{n-i} \\
\leqq & {\left[\sum_{i=0}^{n}\left(\begin{array}{c}
n \\
i
\end{array}\right) p^{i} q^{n-i}\right] \cdot\left[1+\sup _{0 \leqq k \leqq m-1}(a / p)^{k}+\sup _{0 \leqq k \leqq m-1}(b / q)^{k}\right] } \\
\leqq & (p+q)^{n} M,
\end{aligned}
$$

and $\left\|(A+B)^{n}\right\|^{1 / n} \leqq(p+q) M^{1 / n}$, where $M$ is independent of $n$. Thus letting $n \rightarrow \infty$, we have $\|A+B\|_{s} \leqq p+q=\|x\|_{s}+\|y\|_{s}+\varepsilon$.

The next lemma will allow us to conclude $\|x\|_{m}=\|x\|_{s}$ for $x \in T$ from the fact that $\|\cdot\|_{m}$ and $\|\cdot\|_{s}$ annihilate the same elements of $T$. 
LEMma 7.1. Let $f_{1}, f_{2}$ be semi-norms on $T$ such that $f_{1}(x)=0$ if and only if $f_{2}(x)=0$ for $x \in T$. Then there exists $\beta>0$ such that $f_{1}(x) \leqq \beta f_{2}(x)$ for all $x \in T$. Moreover if $f_{1}$ and $f_{2}$ are multiplicative, then $f_{1} \equiv f_{2}$.

Proof. Set $S=\left\{x \in T ; f_{1}(x)=0\right\}$. Then for $r \in R, x, y \in T$, $f_{1}(r x)=r f_{1}(x)=r \cdot 0=0$, and $f_{1}(x+y) \leqq f_{1}(x)+f_{1}(y)=0$, and $S$ is a linear subspace of $E$. Let $Q$ be the quotient space $T / S$. Now for $i=1,2$, $x, y, \in T$ such that $x-y \in S$, we have $\left|f_{i}(x)-f_{i}(y)\right| \leqq f_{i}(x-y)=0$ and thus $f_{i}(x)=f_{i}(y)$. For $i=1,2, x \in T$, setting $x^{\prime}=x+S$, set $f_{i}^{\prime}\left(x^{\prime}\right)=f_{i}(x)$. Then $f_{1}^{\prime}, f_{2}^{\prime}$ are norms on the finite dimensional space $Q$. Hence there exist $0<\alpha<\beta$ such that $\alpha f_{1}^{\prime}(y) \leqq f_{2}^{\prime}(y) \leqq \beta f_{1}^{\prime}(y)$ for all $y \in Q$. Whence $\alpha f_{1}(x) \leqq f_{2}(x) \leqq \beta f_{1}(x)$ for $x \in T$.

Let $x \in T, n \in \omega$, and suppose $f_{1}$ and $f_{2}$ are multiplicative. Then $\alpha f_{1}\left(x^{n}\right) \leqq f_{2}\left(x^{n}\right) \leqq \beta f_{1}\left(x^{n}\right)$ and since $f_{i}\left(x^{n}\right)^{1 / n}=f_{i}(x)$ for

$$
i=1,2, \alpha^{1 / n} f_{1}(x) \leqq f_{2}(x) \leqq \beta^{1 / n} f_{1}(x) \text {. }
$$

Letting $n \rightarrow \infty, \alpha^{1 / n} \rightarrow 1, \beta^{1 / n} \rightarrow 1$, and hence $f_{1}(x) \leqq f_{2}(x) \leqq f_{1}(x)$ and $f_{1} \equiv f_{2}$.

Lemma 7.2. For $x \in T,\|x\|_{s}=\|x\|_{m}$. Moreover for $x \in T$, $\|x\|_{s}=0$ if and only if $x^{p}=0$, where $p$ is the dimension of $E$.

Proof. Set $S_{m}=\left\{x \in T ;\|x\|_{m}=0\right\}, S_{s}=\left\{x \in T ;\|x\|_{s}=0\right\}$, and $S=\left\{x \in T ; x^{p}=0\right\}$. The theorem follows if we show $S=S_{s}=S_{m}$, since if $S_{s}=S_{m}$, from Lemma 7.1, we would have $\|x\|_{s}=\|x\|_{m}$ for all $x \in T$. Now for $\sigma \in S, 0=\|0\|_{s}=\left\|\sigma^{p}\right\|_{s}=\|\sigma\|_{s}^{p}$ and $\sigma \in S_{s}$, and for $x \in S_{s},\|x\|_{m} \leqq\|x\|_{s}=0$, and $x \in S_{m}$. Thus $S \subseteq S_{s} \subseteq S_{m}$.

Let $\sigma \in S_{m}$ and suppose $\sigma^{p} \neq 0$. Consider the sequence

$$
E \supseteqq \sigma(E) \supseteqq \cdots \supseteqq \sigma^{p}(E) \neq\{0\} .
$$

Clearly for some $k \in\{1,2, \cdots, p-1\}, \sigma^{k}(E)=\sigma^{k+1}(E)=\sigma\left[\sigma^{k}(E)\right] \neq\{0\}$ and $\sigma$ is one-to-one on $W=\sigma^{k}(E)$. Since $W$ is invariant under $T$ and $W$ is finite dimensional, there exists $H \in \mathscr{C}$, such that $H \subseteq W$. From Lemma 4.1, $T_{0}=T \mid H$ is a field. Now setting $\sigma_{0}=\sigma \mid H$ and $e_{0}=e \mid H$, there exists $\alpha \in T_{0}$ such that $\sigma_{0} \alpha=e_{0}$. Whence $1=\left\|e_{0}\right\|_{H}=$ $\left\|\sigma_{0} \alpha\right\|_{H} \leqq\left\|\sigma_{0}\right\|_{H}\|\alpha\|_{H}$ and $0<\left\|\sigma_{0}\right\|_{H} \leqq\|\sigma\|_{s}$. But then $\sigma \notin S_{m}$. Thus $\sigma^{p}=0$ and $S_{m} \subseteq S$, and thus $S=S_{m}=S_{s}$.

LEMma 7.3. For $M>1$, there exists $N>0$ such that for $x \in T$, $\|x\| \leqq M$, we have for all $n>p, n \in \omega,\left\|x^{n}\right\| \leqq N\|x\|_{s}^{n-p+1}$.

Proof. Set $S=\left\{\sigma \in T ;\|\sigma\|_{s}=0\right\}$ and let $Q$ be the quotient space 
$T / S$. For $x \in T, x_{0}=x+S$, set $\|x\|_{0}=\left\|x_{0}\right\|=\inf \{\|x+\sigma\| ; \sigma \in S\}$. Then $\|x\|_{0}=0$ if and only if $\|x\|_{s}=0$ for $x \in T$. From Lemma 7.1, there exists $\alpha \geqq 1$, such that $\|x\|_{0} \leqq \alpha\|x\|_{s}$ for $x \in T$. Fix $x \in T$, $\|x\| \leqq M$. Since $x_{0}=x+S$ is finite dimensional, there exists $\bar{x} \in x_{0}$ such that $\|\bar{x}\|=\left\|x_{0}\right\|=\|x\|_{0}$. Set $\sigma=x-\bar{x}$. Then $\sigma \in S$ and from Lemma $7.2, \sigma^{p}=0$.

Let $n \in \omega, n>p$. Then

$$
\|\sigma\|=\|x-\bar{x}\| \leqq\|x\|+\|\bar{x}\| \leqq 2\|x\|
$$

and

$$
\begin{aligned}
\left\|x^{n}\right\| & =\left\|(\bar{x}+\sigma)^{n}\right\|=\left\|\sum_{i=0}^{n}\left(\begin{array}{c}
n \\
i
\end{array}\right) \bar{x}^{n-i} \sigma^{i}\right\|=\left\|\sum_{i=0}^{p-1}\left(\begin{array}{c}
n \\
i
\end{array}\right) \bar{x}^{n-i} \sigma^{i}\right\| \\
& \leqq \sum_{i=0}^{p-1}\left(\begin{array}{c}
n \\
i
\end{array}\right)\|\bar{x}\|^{n-i}\|\sigma\|^{i} \leqq\|\bar{x}\|^{n-p+1} M_{0} \leqq\|x\|_{0}^{n-p+1} M_{0} \\
& \leqq \alpha\|x\|_{s}^{n-p+1} M_{0},
\end{aligned}
$$

where

$$
\begin{aligned}
\alpha M_{0} & =\alpha \sum_{i=0}^{p-1}\left(\begin{array}{c}
n \\
i
\end{array}\right)\|\bar{x}\|^{p-i-1}\|\sigma\|^{i} \leqq \alpha \sum_{i=0}^{p-1}\left(\begin{array}{c}
n \\
i
\end{array}\right)\|x\|^{p-i-1} 2^{i}\|x\|^{2} \\
& \leqq \alpha \sum_{i=0}^{p-1}\left(\begin{array}{c}
n \\
i
\end{array}\right) M^{p-1} 2^{i} \equiv N
\end{aligned}
$$

and thus $\left\|x^{n}\right\| \leqq N\|x\|_{s}^{n-p+1}$.

Theorem 7.2. (Maximal spectral norm theorem). For $f \in F$ such that $\bar{U} \subseteq$ domain $f$, and $x \in U$,

$$
\|f(x)\|_{s} \leqq M=\sup \left\{\|f(t)\|_{s} ; t \in V\right\} .
$$

Proof. We show that $\|f(x)\|_{H} \leqq M$ for all $H \in \mathscr{C}$ and thus that $\|f(x)\|_{s}=\|f(x)\|_{m}=\sup \left\{\|f(x)\|_{H} ; H \in \mathscr{C}\right\} \leqq M$. Let $H \in \mathscr{X}$. We first consider the case when $H$ has dimension greater than one. There are two ways of handling this case. First, applying the fundamental theorem of algebra, we observe that $T \mid H$ under the spectral norm is isomorphic and isometric to $K$. Suitably complexifying $H$ we obtain from classical complex variable theory that $\|f(x)\|_{H} \leqq \sup \left\{\|f(t)\|_{H} ; t \in V \cap[x+H]\right\} \leqq M$.

Proceeding directly we observe, setting $Z=T \mid H$, that $z-\{0\}$ is connected, and hence $\mu(z)=1$ for all $z \in Z-\{0\}$, and thus $Z$ is an orientation preserving family. For $n \in \omega$, from Theorem 5.1.

(1) $\left\|\tilde{f}(x)^{n}\right\| \leqq \sup \left\{\left\|\tilde{f}(t)^{n}\right\| ; t \in V \cap[x+H]\right\}$, where $\tilde{f}(t)=f(t) \mid H$ for $t \in$ domain $f$. From Lemma 7.1, there exists $\alpha \geqq 1$ such that for $x \in T, x_{0}=x \mid H$, we have $\left\|x_{0}\right\| \leqq \alpha\left\|x_{0}\right\|_{s}=\alpha\|x\|_{H I}$. Thus for $n \in \omega, t \in V$, 


$$
\left\|\tilde{f}(t)^{n}\right\| \leqq \alpha\left\|f(t)^{n}\right\|_{H}=\alpha\|f(t)\|_{H}^{n} \leqq \alpha\|f(t)\|_{s}^{n},
$$

and thus from (1),

$$
\begin{aligned}
\left\|\tilde{f}(x)^{n}\right\| & \leqq \alpha \sup \left\{\|f(t)\|_{s}^{n} ; t \in V \cap[x+H]\right\} \\
& \leqq \alpha \sup \left\{\|f(t)\|_{s} ; t \in V\right\}^{n} \\
& =\alpha M^{n}
\end{aligned}
$$

Hence

$$
\|f(x)\|_{H}=\lim _{n \rightarrow \infty}\left\|\tilde{f}(x)^{n}\right\|^{1 / n} \leqq \lim _{n \rightarrow \infty} \alpha^{1 / n} M=M .
$$

We now handle the case when $H$ has dimension one. Suppose $\|f(x)\|_{H}>M$. For $t \in \operatorname{domain} f$, set $g(t)=2 f(t)\left[M+\|f(x)\|_{H}\right]^{-1}$. Then $\|g(x)\|_{I I}>1$, and $M_{0}=\sup \left\{\|g(t)\|_{s} ; t \in V\right\}<1$.

Now from Lemma 7.3, there exists $N>0$ such that $\left\|g(t)^{n}\right\| \leqq$ $N\|g(t)\|_{s}^{n-p+1}$ for $t \in V, n>p, n \in \omega$. Let $\alpha, \beta$ be points of $V$ such that $[\alpha, \beta]=\bar{U} \cap[x+H]$. There exists $y \in[\alpha, \beta], y \neq x$, such that $\|g(t)\|_{I I} \geqq 1$ for all $t \in[x, y]$. Let $A$ be an arc of $V$ with endpoints $\alpha$ and $\beta$. For $t \in[\alpha, \beta]$, there exists $r(t) \equiv R$ such that $|r(t)|=$ $\|g(t)\|_{I I}$ and $[g(t)](y)=r(t) y$ for all $y \in H$. Then for $n>p, n$ even, $n \in \omega, e_{0}=(\beta-\alpha) /\|\beta-\alpha\|$, setting

$$
P=\int_{\alpha_{[\alpha, \beta]}}^{\beta} g(t)^{n} d t \quad \text { and } \quad Q=\int_{\beta_{A}}^{\alpha} g(t)^{n} d t
$$

we have

$$
P+Q=\int_{[\alpha \beta] \cup A} g(t)^{n} d t=0 \text { and }\|P\|=\|Q\|,
$$

and

$$
\|Q\| \leqq \int_{\beta_{A}}^{\alpha}\left\|g(t)^{n}\right\| d s \leqq \int_{\beta_{A}}^{\alpha} N\|g(t)\|_{s}^{n-p+1} d s \leqq N M_{0}^{n-p+1} 2 \pi
$$

and

$$
\begin{aligned}
\|P\| & =\left\|\int_{\alpha[\alpha, \beta]}^{\beta} r(t)^{n} e_{0} d s\right\|=\int_{\alpha[\alpha, \beta]}^{\beta} r(t)^{n} d s \geqq \int_{x_{[x, y]}}^{y} r(t)^{n} d s \geqq \int_{x_{[x, y]}}^{y} 1 d s \\
& =\|y-x\|,
\end{aligned}
$$

where " $d s$ " indicates integration with respect to path length, and thus $0<\|y-x\| \leqq\|P\|=\|Q\| \leqq N M_{0}^{n-p+1}$ for all $n>p$, $n$ even, $n \in \omega$. But then since $M_{0}<1$, we have $\|y-x\|=0$ and $y=x$.

\section{REFERENCES}

1. L. Heffter, Begründing der Functionentheorie auf alten and neuen Wegen, Springer, Berlin, 1955. 
2. K. O. Leland, A characterization of analyticity, Duke Math. J. 33 (1966), 551-565. 3. - Topological analysis of differentiable transformations, Compositio Math., 18 (1967), 189-200.

4. — Algebras of integrable operator valued functions, Preprint, submitted for publication.

5. - Algebras of integrable functions. II, To appear in the Rocky Mountain J. Math.

6. A. J. Macintyre and W. John Wilbur, A proof of the power series expansion without differentiation theorey, Proc. Amer. Math. Soc., 18 (1967), 419-424.

7. F. Riesz and B. Sz.-Nagy, Functional Analysis, Ungar, 1955.

8. G. T. Whyburn, Topological analysis, Second edition, Princeton, 1964.

Received December 2, 1970. This reseach supported by National Science Foundation Grant GP-6311.

ILLINOIS INSTITUTE OF TECHNOLOGY 


\section{PACIFIC JOURNAL OF MATHEMATICS}

\section{EDITORS}

H. SAMELSON

Stanford University

Stanford, California 94305

C. R. HOBBY

University of Washington

Seattle, Washington 98105
J. DugundJI

Department of Mathematics

University of Southern California

Los Angeles, California 90007

RICHARD ARENS

University of California

Los Angeles, California 90024

\section{ASSOCIATE EDITORS}

E. F. BeCKENBACH

B. H. NeumanN

F. WOLF

K. YosHIDA

\section{SUPPORTING INSTITUTIONS}

UNIVERSITY OF BRITISH COLUMBIA

CALIFORNIA INSTITUTE OF TECHNOLOGY

UNIVERSITY OF CALIFORNIA

MONTANA STATE UNIVERSITY

UNIVERSITY OF NEVADA

NEW MEXICO STATE UNIVERSITY

OREGON STATE UNIVERSITY

UNIVERSITY OF OREGON

OSARA UNIVERSITY
UNIVERSITY OF SOUTHERN CALIFORNIA STANFORD UNIVERSITY

UNIVERSITY OF TOKYO

UNIVERSITY OF UTAH

WASHINGTON STATE UNIVERSITY

UNIVERSITY OF WASHINGTON

AMERICAN MATHEMATICAL SOCIETY

NAVAL WEAPONS CENTER

Printed in Japan by International Academic Printing Co., Ltd., Tokyo, Japan 


\section{Pacific Journal of Mathematics}

\section{Vol. 40, No. $1 \quad$ September, 1972}

Alex Bacopoulos and Athanassios G. Kartsatos, On polynomials

approximating the solutions of nonlinear differential equations........

Monte Boisen and Max Dean Larsen, Prüfer and valuation rings with zero

divisors ..........................................

James J. Bowe, Neat homomorphisms

David W. Boyd and Hershy Kisilevsky, The Diophantine equation

$$
u(u+1)(u+2)(u+3)=v(v+1)(v+2) \ldots \ldots \ldots \ldots \ldots \ldots \ldots
$$

George Ulrich Brauer, Summability and Fourier analysis ...............

Robin B. S. Brooks, On removing coincidences of two maps when only one,

rather than both, of them may be deformed by a homotopy ............

Frank Castagna and Geert Caleb Ernst Prins, Every generalized Petersen

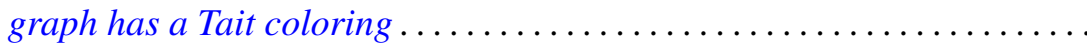

Micheal Neal Dyer, Rational homology and Whitehead products ..........

John Fuelberth and Mark Lawrence Teply, The singular submodule of a

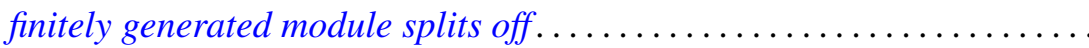

Robert Gold, $\Gamma$-extensions of imaginary quadratic fields ............ 83

Myron Goldberg and John W. Moon, Cycles in k-strong tournaments.......

Darald Joe Hartfiel and J. W. Spellmann, Diagonal similarity of irreducible

matrices to row stochastic matrices...............

Wayland M. Hubbart, Some results on blocks over local fields ..

Alan Loeb Kostinsky, Projective lattices and bounded homomorphisms....

Kenneth O. Leland, Maximum modulus theorems for algebras of operator

valued functions ...

Jerome Irving Malitz and William Nelson Reinhardt, Maximal models in the

language with quantifier "there exist uncountably many" ..

John Douglas Moore, Isometric immersions of space forms in space

forms.

Ronald C. Mullin and Ralph Gordon Stanton, A map-theoretic approach to

Davenport-Schinzel sequences ....................

Chull Park, On Fredholm transformations in Yeh-Wiener space. .

Stanley Poreda, Complex Chebyshev alterations ..............

Ray C. Shiflett, Extreme Markov operators and the orbits of Ryff. ...

Robert L. Snider, Lattices of radicals .....................

Ralph Richard Summerhill, Unknotting cones in the topological

category ................................

Charles Irvin Vinsonhaler, A note on two generalizations of $\mathrm{QF}-3 \ldots \ldots 229$

William Patterson Wardlaw, Defining relations for certain integrally

parameterized Chevalley groups...................

William Jennings Wickless, Abelian groups which admit only nilpotent

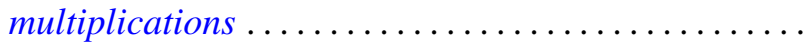

